\title{
A sinister black finding in the stomach
}

Wladyslaw Januszewicz, Pippa Corrie, Hongxiang Liu, James Chan, Rebecca C Fitzgerald, Massimiliano di Pietro

MRC Cancer Unit, University of Cambridge (W Januszewicz MD, RC Fitzgerald PhD, M di Pietro MD), Department of Histopathology, Cambridge University Hospitals NHS Foundation Trust and Cambridge University Medical School $(\mathrm{H}$ Liu PhD, J Chan BM), Cambridge Cancer Centre, Cambridge University Hospitals NHS Foundation Trust and Cambridge University Medical School (P Corrie PhD), Cambridge, UK. Department of Gastroenterology, Hepatology and Clinical Oncology, Medical Centre for Postgraduate Education, Warsaw, Poland (W Januszewicz MD).

\section{Correspondence to:}

Dr Wladyslaw Januszewicz

MRC Cancer Unit, University of Cambridge, Cambridge CB2 0XZ, UK

w.j264@mrc-cu.cam.ac.uk

A 68-year old woman undergoing a routine surveillance endoscopy-part of her follow-up for a Barrett's oesophagus - was found to have a solitary, $4 \mathrm{~mm}$, black lesion in the gastric body (Figure A). Biopsies were taken which showed mucosa typical of the gastric body with heavily pigmented cells within the lamina propria infiltrating around the crypts without destroying them (Figure B). Immunohistochemical examination of biopsied tissue section showed positive staining for melanoma associated antigens using antibodies including Melan-A, HMB-45, MITF, and SOX-10. Her medical history included the enucleation of her left eye 13 years earlier to remove a primary uveal melanoma; she had been discharged from routine follow-up. We then decided to carry out another gastroscopy to take deeper gastric biopsies. These showed infiltration of the muscularis mucosae by melanocytes with mild atypia. A full-body CT scan showed multiple lesions in her liver which on subsequent MRI were confirmed to be melaninladen metastases (Figure C). Gene mutation panel testing on the gastric biopsy specimens and on the ocular specimens taken at the time of the eye operation 13 years earlier, showed the same oncogenic mutation: Gln209Leu in GNA11. Tests for the common cutaneous melanoma mutations in $B R A F, N R A S$, and KIT genes were negative. The patient was enrolled in a clinical trial testing a combination of selumetinib and paclitaxel chemotherapy for metastatic uveal melanoma.

Uveal melanoma is a rare cancer representing less than 5\% of all melanomas; it has a nearly 50-fold lower incidence rate compared with that of cutaneous melanomas. It is an aggressive disease predominantly spreading to the liver in up to $50 \%$ of patients. Median survival with liver metastases is under 6 months. Currently, there are no effective non-surgical treatments for metastatic uveal melanoma. Surveillance and monitoring of the liver-after treatment of the primary lesion-is recommended so that patients can receive early locoregional treatments. The length of time patients should be followed-up is not established in current clinical guidelines. Uveal melanoma is associated with an almost linear continuation of recurrence over time and beyond 10 years without a plateau in risk of recurrence over time. Thus, late recurrences are not uncommon: 5- and 10-year cumulative metastasis rate is $25 \%$ and $34 \%$, respectively. Cutaneous and uveal melanomas are biologically distinct. Uveal melanomas lack the typical 
cutaneous melanoma-associated mutations in the BRAF, NRAS and NF1 genes, but have somatic mutations in the GNA11 and QNAQ genes in around $90 \%$ of cases-in cutaneous melanomas mutations in these genes are found in less than $10 \%$ of cases. Mutations in these genes activate the MAP kinase oncogenic pathway, providing a rationale for using MEK1/2 inhibitors, selumetinib for example, as agents in clinical trials results - although to date the results have been disappointing. Functionally activating mutations in GNAQ and GNA11, as well as in CYSLTR2 and PLCB4, leads to the subsequent activation of pathways - which include the PI3kinase and Yap/Hippo pathways - downstream beyond the MAP kinase pathway. These may offer potential novel therapeutic targets. Genetic profiling of primary uveal melanomas has some prognostic value. For example, inactivating mutations in the $B A P 1$ gene-found in approximately $50 \%$ of cases - are highly likely to metastasise, whereas mutations in the SF3B1 and EIFIAX genes, occurring in approximately $19 \%$ and $24 \%$ of uveal melanoma cases respectively, are less likely to metastasise.

\section{Contributors}

WJ and PC searched the literature and analysed the results. RCF and MdP cared for the patient. WJ, PC, RCF and MdP wrote the report. WJ and MdP did the endoscopy and provided the images. HL did the genetic analysis. JC did the histological assessment and provided the images. All authors critically revised the report. Written consent for publication was obtained from the patient.

\section{(C) 2019 Elsevier Ltd. All rights reserved.}

Figure: Surprise black finding at routine endoscopy for Barrett's oesophagus (A) Solitary, $4 \mathrm{~mm}$, black lesion on the greater curvature of the mid-gastric body seen at endoscopy. (B) Typical gastric body mucosa shows heavily pigmented cells within the lamina propria infiltrating around the crypts (haematoxylin and eosin stain). Original magnification $\mathrm{x}$ 5. (C) Contrast-enhanced MRI shows multiple T1-hyperintense lesions in the liver-the largest measured up to $2 \mathrm{~cm}$. The lesions were hypointense on T2-weighted and diffusion-weighted imaging.
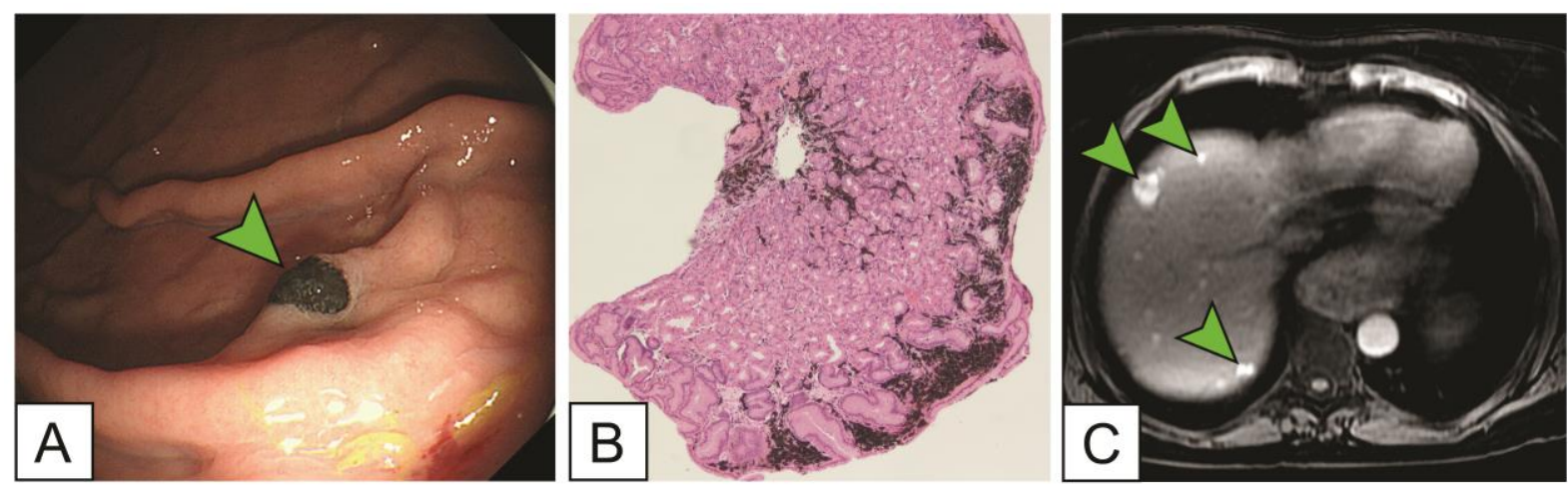